American J. of Engineering and Applied Sciences 4 (2): 288-293, 2011

ISSN 1941-7020

(C) 2011 Science Publications

\title{
An Effective Approach for Benchmarking Implementation
}

\author{
B.M. Deros, J. Tan, M.N. Ab. Rahman and N.A.Q.M. Daud \\ Department of Mechanical and Materials Engineering, \\ Faculty of Engineering and Built Environment, \\ University Kebangsaan Malaysia, Bangi Selangor, 43600, Malaysia
}

\begin{abstract}
Problem statement: The purpose of this study is to present a benchmarking guideline, conceptual framework and computerized mini program to assists companies achieve better performance in terms of quality, cost, delivery, supply chain and eventually increase their competitiveness in the market. The study begins with literature review on benchmarking definition, barriers and advantages from the implementation and the study of benchmarking framework. Approach: Thirty respondents were involved in the case study. They comprise of industrial practitioners, which had assessed usability and practicability of the guideline, conceptual framework and computerized mini program. Results: A guideline and template were proposed to simplify the adoption of benchmarking techniques. A conceptual framework was proposed by integrating the Deming's PDCA and Six Sigma DMAIC theory. It was provided a step-by-step method to simplify the implementation and to optimize the benchmarking results. A computerized mini program was suggested to assist the users in adopting the technique as part of improvement project. As the result from the assessment test, the respondents found that the implementation method provided an idea for company to initiate benchmarking implementation and it guides them to achieve the desired goal as set in a benchmarking project. Conclusion: The result obtained and discussed in this study can be applied in implementing benchmarking in a more systematic way for ensuring its success.
\end{abstract}

Key words: Benchmarking framework, continual improvement, benchmarking implementation, conceptual framework, industrial practitioners, conceptual framework, assessed usability, deming's PDCA, six sigma, implementing benchmarking

\section{INTRODUCTION}

According to Lucertini (1995) there are many definitions for the term "benchmarking" that are based on the idea of evaluating performance. Hornby (2002) dictionary had defined benchmarking as standard example and point of reference for making comparisons. However, benchmarking in general can be defined as key themes that include measurement, comparison and identification of best practices, implementation and improvement (Anand and Kodali, 2008). Ribeiro and Cabral (2006) had found that benchmarking give benefits to companies especially in the metal casting industry but it is a timeconsuming tool and demands continuous commitment of the topmanagers. Also, (Fry et al., 2005), claimed that benchmarking was identified as the most used performance improvement technique for both airlines and airports. From these cases, it obviously indicates that benchmarking had played a significant role in assisting companies from different fields to grow and become successful. Furthermore, benchmarking has also become an integral part of organizational improvement methodology (Boulter, 2003).

On the other hand, Asrofah et al. (2010) stated that there are many companies, which emphasize on the importance of benchmarking, however, not many companies understand well enough about benchmarking. The lack of a holistic understanding of benchmarking is one of the main causes that make it is difficult for some companies to employ the tools effectively. According to a study conducted by Amaral and Sousa (2009), the barriers that constrains the company from implementing benchmarking consists of organizational barriers (people, culture and context), benchmarking project management barriers (planning and implementation, leadership and business pressures) and benchmarking data barriers (difficulty to access/compare data). As the result, formalizing the benchmarking model with methods and tools would be one of the best ways to overcome the obstacles (Buyukozkan and Maire, 1998).

Modern benchmarking practice and theory in business did not come into being until the pioneering

Corresponding Author: B.M. Deros, Department of Mechanical and Materials Engineering, Faculty of Engineering and Built Environment, University Kebangsaan Malaysia, Bangi Selangor, 43600, Malaysia 
work of Robert C. Camp and his team at Xerox in the early 1980s (Shen et al., 2000; Zairi, 1994). Shen et al. (2000) further explained that as a business improvement and quality tool, benchmarking has became widely covered in literature and broadly applied in practice. Camp (1989) had developed the benchmarking wheel which adopted the PDCA (plan, do, check and act) cycle. Throughout these years, the frameworks are developed generally based on Deming's PDCA theory and the studies that evolved from Camp's benchmarking wheel. Some of the authors had further developed the phase and steps to provide a better understanding on benchmarking concept and eventually implemented the technique with effective result. This can be seen in the framework that was developed (Zairi, 1994; Ahmed and Hassan, 2003; Ribeiro and Cabral, 2006; Deros et al., 2006).

The main objective of this study is to propose a guideline to simplify benchmarking process. It will suggest the method for the user to achieve the goal of benchmarking projects. Deros et al. (2006) argues that benchmarking encourages a company to become more open to new methods, ideas, processes and practices to improve effectiveness, efficiency and performance. Towards the implementation, simplicity is one of the significant factors to be emphasized so that the users are not confused along the way of implementing benchmarking. A template will also be recommended and it serves as the core activity to benchmark the data. From here, the users will obtain the benchmarking results and start to plan for the continual improvement activities. The structure of this study will discuss about what is benchmarking, the advantages of implementation and framework study. These will then be followed by the guideline for benchmarking implementation and future research is suggested in the conclusion of this study.

\section{MATERIALS AND METHODS}

This section discussed about the methodology applied in the research. Thirty respondents were involved in the case study. They comprise of thirty industrial practitioners, which are currently involved in performing benchmarking activities in their daily work. Their tasks are to assessed usability and practicability of the proposed guideline, conceptual framework and computerized mini program.

Deros et al. (2006) had defined framework as a set of simplified theoretical principles and practical guidelines to carry out benchmarking implementation and adoption, which can enhance the chances of success that are easy to understand, efficient and can be implemented at reasonable costs and time. In addition to that, it is important to have a framework as a guideline to adopt benchmarking technique as a tool for continuous improvement activities. The proposed framework was developed based on Deming's PDCA and six sigma problem solving methods.

Besides the framework, a computerized benchmarking program was designed to assist the user to benchmark the data. The design of the computerized benchmarking program was based on the generic product development process concept developed by Ulrich and Eppinger (1999) which comprise of 6 phases as follows:

- $\quad$ Phase 0: Planning

- $\quad$ Phase 1: Concept development

- $\quad$ Phase 2: System-level design

- $\quad$ Phase 3: Detail design

- $\quad$ Phase 4: Testing and refinement

- $\quad$ Phase 5: Production ramp-up

The development of the benchmarking software had made it possible to encompass Phase 0 until Phase 4 within the program itself. An assessment test was carried out by 30 respondents to understand on the usability, practicability and effectiveness of the computerized benchmarking program.

\section{RESULTS AND DISCUSSION}

According to Razmi et al. (2000) choosing the right benchmarking methodology is an essential key in making benchmarking a success. The main concept of the framework is initiated by Deming's PDCA theory. Ahmed and Hassan (2003) argued that a systematic approach can provide significant benefits in the long run. Deming's plan-do-check-act (PDCA) is an excellent technique in monitoring and problem solving for continuous quality improvement where individual's brilliant ideas can be accommodated. As such, it is suitable to apply Deming's PDCA approach to guide the user with a proper and systematic way of implementation. However, it is not adequate to apply only PDCA approach. To increase the benchmarking effectiveness, Six Sigma's DMAIC theory is incorporated into the framework. Based on Tjahjono et al. (2010) DMAIC is a problem-solving method which aims at process improvement. Nevertheless, DMAIC is actually close to the original process that Deming defined which generated PDCA in Japan (Watson and DeYong, 2010). With the combination of these two approaches, a systematic step-by-step method was developed to be used as guidance for the benchmarking implementation. Users are recommended to adopt this framework in performing benchmarking process and eventually use the benchmarking results to determine the appropriate improvement activities. Figure 1 the proposed conceptual framework for benchmarking technique implementation. 
Am. J. Engg. \& Applied Sci., 4 (2): 288-293, 2011

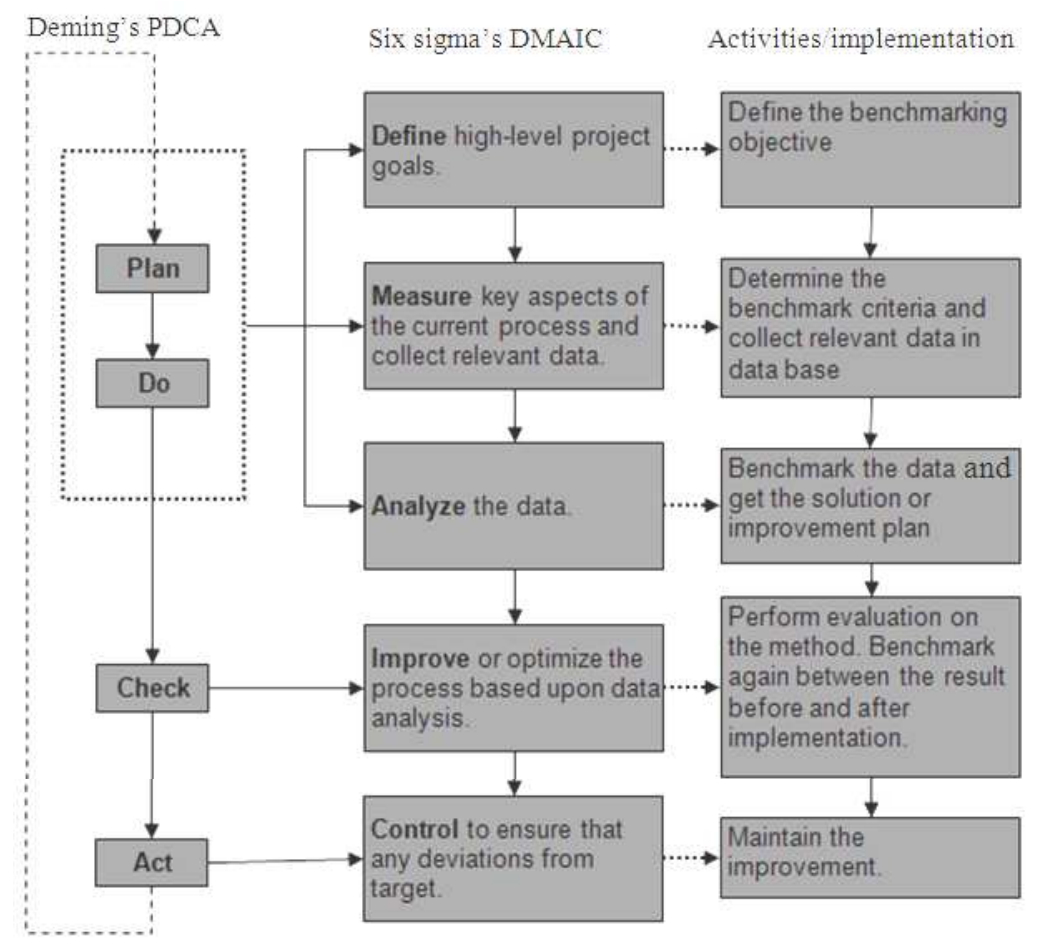

Fig. 1: Benchmarking technique implementation framework

PDCA-"Planning stage": By following the steps in the framework as shown in Fig. 1, the companies firstly need to plan and execute a benchmarking project. The goal or objective of the project needs to be defined at the beginning stage so that it would not divert from the target. All the details are recommended to be put into a template, so that the method could be standardized. Standardization is important to maintain a systematic implementation. According to Ahmed and Rafiq (1998) as quoted from Watson and DeYong (2010), the key questions to ask when users initiate a benchmarking project are as follows:

- What should we benchmark

- Whom should we benchmark

- How do we perform the process

- How do they perform the process

With the clarification of these research questions, the users could have a better understanding on the scope of the benchmarking project execution.

PDCA, doing stage: After goal setting, the users can measure their current performance by collecting the benchmarking data. It is recommended that the data is stored in a database system. A good database system is not only able to provide a good traceability. It also caters a visibility comparison between the data. With the benchmarking database, the user can sort the data based on the priority of the criteria. In this case, let the data "talk and tell" what is the appropriate approach in the next step. Also, the improvement activities could be generated from the benchmarking results. Furthermore, if it is a problem solving issue, the user can analyze and determine the potential root cause and the appropriate corrective action by using the benchmarking data. For example, to resolve the major defect in the production floor, the user shall collect and benchmark the defect quantity. From the benchmarking process, the data will "tell" the major defect that significantly cause low yield to the process. Finding root causes and corrective actions are the next steps after identifying the major defect.

Checking stage: The benchmarking process not only stops after implementing the improvement activities or corrective actions but verification of the effectiveness is needed. Evaluation needs to be carried out to check the effectiveness of the method. In this stage, the benchmarking approach is used to benchmark the results before and after the improvement activities have been implemented. A report shall be generated to capture the improvement. 

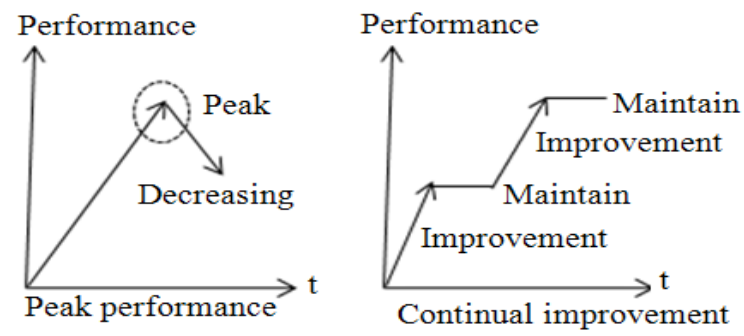

Fig. 2: The difference between peak performance and continual improvement

Acting stage: At this stage, if the users find that the results show positive improvement, they have to maintain the improvement activities to ensure the continual improvement. Or vice versa, the company needs to adopt other method in order to achieve a better performance. If so, the benchmarking process needs to start all over again under the Deming's PDCA as shown in the conceptual framework. The framework is emphasizing on continual improvement rather than peak performance. Figure 2 illustrates the comparison between peak performance and continual improvement attribute. A peak performance that shows an uptrend of the improvement, however, at a certain point of time, will decrease drastically. Continual improvement indicates a trend of improvement and maintains it from time to time. Once the improvement is maintained, it will improve again by other improvement activities using the cycle of Deming's PDCA.

Computerized benchmarking program: Towards the implementation by using this guideline we need a computerized benchmarking program to ensure the traceability of the data and the analysis will be captured from the project. The program shall contain all the information for the benchmarking project. "Criteria sorting" is used to prioritize the criteria based on the significance of the criteria. From the criteria, the users can enter either the rating or the actual number of the benchmarking model. For example as shown in Fig. 3, the users can enter the actual cost in the "cost" column. For the other criteria like "quality", "service" and "delivery", the users can rate each of the models and put the relative rate into the tables. Arranging by ascending or descending order for each column is needed as the last stage to analyze the benchmarking data. By using sorting method, the users could clearly see which model has the best performance. Furthermore, if the users would like to assess self performance, they can enter their performance into the table. The program will tell the ranking of the performance. From benchmarking analysis, the users could understand what is missing in current performance and initiate the improvement plan so that they could be able to achieve better results. Figure 3 illustrates the template of the benchmarking system.

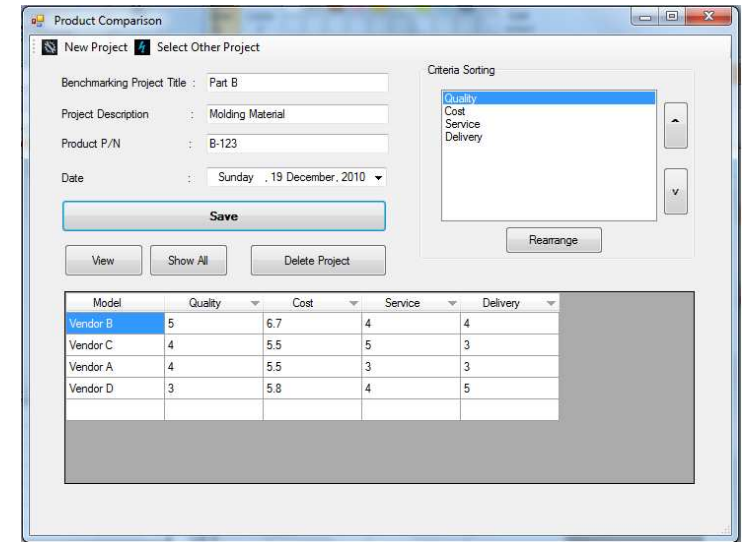

Fig. 3: Computerized benchmarking program

An assessment had been carried out by 30 respondents in the industry who had applied benchmarking as the improvement tools and they agreed that benchmarking is important to understand their strengths and weaknesses. However, not many of them implemented benchmarking in a systematic way. With respect to the benchmarking guide, framework and mini computerized benchmarking program, $80 \%$ of them agreed that it helps them in the benchmarking implementation process. In other words, the respondents had shown much interest in the systematic guidelines, framework and computerized benchmarking program. From the rating, the respondents agreed that it is very useful to use a database concept for comparing benchmarking data. The program had met the user requirements and their expectations. On the other hand, there is room for improvement. The users wish that there is a summary that could be generated by the program after the benchmarking data has been analyzed. This could simplify the report generation and also the report could be used as presentation material. Lastly, with the presence of this program, the authors hope that it could assist users further in obtaining the necessary results and optimizing the benchmarking outcome.

\section{CONCLUSION}

Benchmarking implementation has not been an easy task due to the lack of benchmarking understanding and systematic utilization method. In the overall view of benchmarking implementation, the case study results indicate that the industry had applied a lot of comparison activities to obtain better results. However, the users' understanding of benchmarking concepts and techniques is still lacking. In this case, a well organized framework simply plays an important role to guide the users in implementation and performance optimization. In order to maximize the 
benchmarking results, the users should be given more training and exposure so that they would be well equipped with the knowledge and carry out the implementation effectively.

Benchmarking approach is providing a persuasive data and results of the project. Furthermore, it gives a better understanding on the current situation and also enables the user to take a suitable action to improve the situation. Benchmarking technique can be used to identify the strengths and weaknesses of the company. Deming's PDCA and six sigma's DMAIC theory are integrated to design a framework to ensure that the implementation is systematic and in a proper way. By using the conceptual framework, the users will see a clearer picture on the implementation and eventually obtain the desired result as per defined at the early stage of a benchmarking project.

The proposed guideline is not aimed as a compulsory tool for benchmarking implementation. However, it is a recommended tool that takes advantage of a statistical analysis from database to understand the current performance and use the benchmarked data as a platform for generating improvement plan. Thus, this will ensure that continuous improvement activities are in place all the time. Furthermore, the proposed framework is not only applicable in certain area, but it is usable in all the area or plant-wide. With the assistance of the framework and computerized benchmarking program, the users could have a better understanding on the concept of benchmarking technique so that they understand and capable on how to initiate a benchmarking project and carry out the implementation process.

\section{ACKNOWLEDGMENT}

The researchers would like to acknowledge the financial contributions both from Ministry of Science, Technology and Innovation Malaysia and Ministry of Higher Education Malaysia for their research grants.

\section{REFERENCES}

Ahmed, P.K. and M. Rafiq, 1998. Integrated benchmarking a holistic examination of select techniques for benchmarking analysis. Benchmarking for quality manage. Technology, 5: 225-242. DOI: 10.1108/14635779810234802

Ahmed, S. and M. Hassan, 2003. Survey and case investigations on application of quality management tools and techniques in SMIs. Int. J. Quality Reliability Manage., 20: 795-826. DOI: 10.1108/02656710310491221
Amaral, P. and R. Sousa, 2009. Barriers to internal benchmarking initiatives. An empirical investigation. Benchmarking. Int. J., 16: 523-542. DOI: $10.1108 / 14635770910972441$

Anand, G. and R. Kodali, 2008. Benchmarking the benchmarking models. Benchmarking. Int. J., 15: 257-291. DOI: $10.1108 / 14635770810876593$

Asrofah, T., S. Zailani and Y. Fernando, 2010. Best practices for the effectiveness of benchmarking in the Indonesian manufacturing companies. Benchmarking. Int. J., 17: 115-143. DOI: 10.1108/14635771011022343

Boulter, L., 2003. Legal issues in benchmarking. Benchmarking. Int. J., 10: 528-537. DOI: 10.1108/14635770310505166

Buyukozkan, G. and J.L. Maire, 1998. Benchmarking process formalization and a case study. Benchmarking for quality manage. Technology, 5: 101-125. DOI: $10.1108 / 14635779810212356$

Camp, R.C., 1989. Benchmarking: The Search for Industry Best Practices that Lead to Superior Performance. 1st Edn., ASQC Quality Press, Milwaukee, WI., ISBN-13: 978-0527916350, pp: 299.

Deros, B.M., S.M. Yusof and A.M. Salleh, 2006. A benchmarking implementation framework for automotive manufacturing SMEs benchmarking. Int. J., 13: 396-430. DOI: $10.1108 / 14635770610676272$

Fry, J., I. Humphreys and G. Francis, 2005. Benchmarking in civil aviation. Some empirical evidence. Benchmarking. Int. J., 12: 125-137. DOI: $10.1108 / 14635770510593077$

Hornby, A.S., 2002. Oxford Advance Learner's English-Chinese Dictionary. 4th Edn., The Commercial Press, New York, ISBN-10: 7100034159, pp: 2016.

Lucertini, M., 1995. Integration of benchmarking and benchmarking of integration. Int. Produc. Econ., 38: 59-71. DOI: 10.1016/0925-5273(95)99064-C

Razmi, J., M. Zairi and Y.F. Jarrar, 2000. The application of graphical techniques in evaluating benchmarking partners benchmarking. Int. J., 7: 304-314. DOI: $10.1108 / 14635770010378936$

Ribeiro, L.M.M. and J.A.S. Cabral, 2006. A benchmarking methodology for metalcasting industry. Benchmarking. Int. J., 13: 23-35. DOI: 10.1108/14635770610644556

Shen, X.X., K.C. Tan and M. Xie, 2000. Benchmarking in QFD for quality improvement benchmarking. Int. J., 7: 282-291. DOI: $10.1108 / 14635770010378918$ 
Tjahjono, B.P., V. Ball, I. Vitanov, C. Scorzafave and J. Nogueira et al., 2010. Six sigma. A literature review. In. J. Lean Six Sigma., 1: 216-233. DOI: 10.1108/20401461011075017

Ulrich, K. and S. Eppinger, 1999. Product Design and Development. 2nd Edn., McGraw Hill/Irwin, New York, ISBN: 10: 007229647X, pp: 384.
Watson, G.H. and C.F. DeYong, 2010. Design for six sigma. caveat emptor. Int. J. Lean Six Sigma, 1: 66-84. DOI: $10.1108 / 20401461011033176$

Zairi, M., 1994. Benchmarking the best tool for measuring competitiveness. Benchmarking for quality manage. Technology, 1: 1315-3036. DOI: $10.1108 / 14635779410056859$ 